\title{
ON THE PROJECTION OF A SELFADJOINT OPERATOR
}

\author{
BY WILLIAM STENGER ${ }^{1}$
}

\author{
Communicated by J. B. Diaz, October 13, 1967
}

In the present note some new properties of selfadjoint operators are given. These results came to light in our investigation of Weinstein's new maximum-minimum theory of eigenvalues [1], [2], but may be of some general interest by themselves, since they do not refer explicitly to eigenvalues. For the theory of unbounded operators, as used here, see the recent book of Goldberg [3].

Let $T$ be any selfadjoint linear operator on a dense subspace $\mathscr{D}$ of a Hilbert space $\mathfrak{F}$. Let $\mathcal{Y}$ be any closed subspace of $\mathfrak{F}$, let $Y$ be the projection operator on to $\mathcal{y}$, and let $x=y^{\perp}$ be the orthogonal complement of $y$ in $\mathfrak{K}$. Then we have the following results.

Lemma 1. If $X$ is finite-dimensional, then the operator $T_{0}=Y T Y$ is selfadjoint.

Proof. Let $D_{0}$ denote the domain of $T_{0}$. Clearly $D_{0}=x \oplus$ (D’Y). The fact that $D_{0}$ is dense in $\mathcal{H}$ follows from a lemma of Gohberg and Krein [4], see also Goldberg [3, p. 103], which states that the intersection of a dense subspace and a closed subspace having finite deficiency is dense in the latter. Since $T_{0}$ is symmetric, it suffices to show that $D_{0}=D_{0}^{*}$, where $D_{0}^{*}$ denotes the domain of the adjoint of $T_{0}$. First of all, $u \in D_{0}$ implies that $Y u \in D=D^{*}$. Therefore $(T v, Y u)$ is continuous for all $v \in D$. In particular, $(T Y v, Y u)$ is continuous for all $Y v \in D$, so that $(Y T Y v, u)$ is continuous for all $v \in D_{0}$. This means that $u \in D_{0}^{*}$, and therefore $\mathscr{D}_{0} \subset D_{0}^{*}$. On the other hand, let $u \in D_{0}^{*}$. Then $(Y T Y v, u)=(T Y v, Y u)$ is continuous for all $v \in D_{0}$ or equiva-

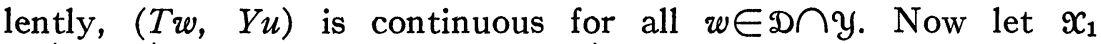
$=\{x \in x \mid \exists y \in \mathcal{Y}$ for which $x+y \in \mathbb{D}\} . x_{1}$ is clearly a subspace (not necessarily proper) of $x$ and therefore finite-dimensional. ${ }^{2}$ Let $x_{1}$, $x_{2}, \cdots, x_{s}$ be any orthonormal basis for $x_{1}$ and let $y_{1}, y_{2}, \cdots, y_{s}$ be any elements in $y$ such that $z_{j}=x_{j}+y_{j} \in D, j=1,2, \cdots, s$. Let $Z=\operatorname{sp}\left\{z_{1}, z_{2}, \cdots, z_{s}\right\}$. It is easy to see that $D=Z+(D \cap Y)$. Now let $W_{0}=\mathscr{D} \cap \mathcal{Y}$ and

$$
\mathscr{W}_{j}=\operatorname{sp}\left\{z_{1}, z_{2}, \cdots, z_{j}\right\}+\mathscr{W}_{0}, \quad j=1,2, \cdots, s .
$$

${ }^{1}$ Research partially sponsored by the Air Force Office of Scientific Research, Office of Aerospace Research, United States Air Force, under AFOSR Grant 130667.

${ }^{2}$ It can be shown that $X_{1}=X$ but this fact is not needed for the proof of the lemma. 
We have already shown that $(T w, Y u)$ is continuous on $w_{0}$. Suppose that $(T w, Y u)$ is continuous on $w_{k}, 0 \leqq k<s$. Let $w_{k+1}$ denote any element in $w_{k+1}$. Then we can write $w_{k+1}=\alpha z_{k+1}+w_{k}$, where $w_{k} \in w_{k}$. By construction, $w_{k}$ is orthogonal to $x_{k+1}$ and therefore

$$
\begin{aligned}
\left|\left(z_{k+1}, w_{k}\right)\right| & =\left|\left(x_{k+1}+y_{k+1}, w_{k}\right)\right|=\left|\left(y_{k+1}, w_{k}\right)\right| \\
& \leqq\left\|y_{k+1}\right\|\left\|w_{k}\right\|=\left[\| z_{k+1}||^{2}-1\right]^{1 / 2}\left\|w_{k}\right\| \\
& =\left[1-1 /\left\|z_{k+1}\right\|^{2}\right]^{1 / 2}\left\|z_{k+1}\right\|\left\|w_{k}\right\| \\
& \leqq\left(1-\epsilon_{k+1}\right)\left\|z_{k+1}\right\|\left\|w_{k}\right\|
\end{aligned}
$$

for some $\epsilon_{k+1}, 0<\epsilon_{k+1}<1$, where $\epsilon_{k+1}$ depends only on $x_{k+1}$ and $y_{k+1}$. Now we have by direct computation,

$$
\begin{aligned}
\left\|w_{k+1}\right\|^{2} & \geqq|\alpha|^{2}\left\|z_{k+1}||^{2}-2|\alpha|\left|\left(w_{k}, z_{k+1}\right)\right|+\right\| w_{k} \|^{2} \\
& \geqq|\alpha|^{2}\left\|z_{k+1}||^{2}-2|\alpha|\left(1-\epsilon_{k+1}\right)\right\| z_{k+1}\|\| w_{k}\|+\| w_{k} \|^{2} \\
& =\left[|\alpha|\left\|z_{k+1}\right\|-\left\|w_{k}\right\|\right]^{2}+2 \epsilon_{k+1}|\alpha|\left\|w_{k}\right\|\left\|z_{k+1}\right\| .
\end{aligned}
$$

This inequality (1) means that

$$
2|\alpha|\left\|w_{k}\right\||| z_{k+1}\|\leqq\| w_{k+1}||^{2} / \epsilon_{k+1}
$$

and

$$
|\alpha|^{2}\left\|z_{k+1}\right\|^{2}-2|\alpha|\left\|w_{k}\right\|\left\|z_{k+1}\right\|+\left\|w_{k}\right\|^{2} \leqq\left\|w_{k+1}\right\|^{2} .
$$

Combining the inequalities (2) and (3) we see that

$$
|\alpha|{ }^{2}\left\|z_{k+1}||^{2}+\right\| w_{k}\left\|^{2} \leqq\left(1+1 / \epsilon_{k+1}\right)\right\| w_{k+1} \|^{2} .
$$

Now letting $\eta_{k+1}=\left(1+1 / \epsilon_{k+1}\right)^{1 / 2}$ we obtain from (4) the inequalities

$$
\begin{aligned}
|\alpha|\left\|z_{k+1}\right\| & \leqq \eta_{k+1}\left\|w_{k+1}\right\|, \\
\left\|w_{k}\right\| & \leqq \eta_{k+1}\left\|w_{k+1}\right\| .
\end{aligned}
$$

Therefore, from the finite-dimensionality of $\mathcal{Z}$ and the continuity of $(T w, Y u)$ on $w_{k}$, we have

$$
\begin{aligned}
\left|\left(T w_{k+1}, Y u\right)\right| & \leqq|\alpha|\left|\left(T z_{k+1}, Y u\right)\right|+\left|\left(T w_{k}, Y u\right)\right| \\
& \leqq|\alpha| \gamma_{k+1}|| z_{k+1}||+\beta_{k}|| w_{k} \| .
\end{aligned}
$$

Using the inequalities (5) in (6) we obtain

$$
\left|\left(T w_{k+1}, Y u\right)\right| \leqq \beta_{k+1}|| w_{k+1} \|,
$$

where $\beta_{k+1}=\eta_{k+1}\left(\gamma_{k+1}+\beta_{k}\right)$, and therefore $(T w, Y u)$ is continuous on $W_{k+1}, k=0,1, \cdots, s-1$.

Since $D=Z+(D \cap Y)=W_{s}$, this means that $Y u \in D^{*}=\mathscr{D}$. 


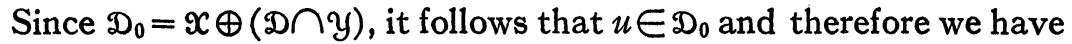
$D_{0}=D_{0}^{*}$.

For an application of the above result see [6].

Q.E.D.

Lemma 2. If $X$ is a subspace of $D$, then the operator $T_{0}=Y T Y$ is selfadjoint.

Proof. Since $X$ is contained in $D$, we have $D=X \oplus D \cap Y=D_{0}$, and therefore $D_{0}$ is dense in 3 C. As in Lemma 1 , it suffices to show that $D_{0}=D_{0}^{*}$. The same argument used above proves that $D_{0} \subset D_{0}^{*}$. Now suppose that $u \in D_{0}^{*}$. Then $(Y T Y v, u)=(T Y v, Y u)$ is continuous for all $v \in \mathscr{D}_{0}$, which means that

$$
|(T w, Y u)| \leqq \alpha\|w\|
$$

for all $w \in D \cap y$. Since $T$ is a closed operator and $X$ is a closed subspace of $D$, it follows from the closed graph theorem that $T$ is continuous on $X$. Therefore we have

$$
|(T x, Y u)| \leqq \beta\|x\|
$$

for all $x \in \mathscr{X}$. Let $\gamma=2 \max \{\alpha, \beta\}$. For any $z \in D=\mathscr{X} \oplus D \cap y$ we can write $z=x+w$ where $x \in X$ and $w \in D \cap Y$. Applying the inequalities (7) and (8) we obtain

$$
\begin{aligned}
|(T z, Y u)| & \leqq|(T x, Y u)|+|(T w, Y u)| \leqq \beta\|x\|+\alpha\|w\| \\
& \leqq \gamma\|x+w\| .
\end{aligned}
$$

This means that $Y u \in D^{*}=D^{\prime}=D_{0}$, and therefore $u \in D_{0}$. Q.E.D.

REMARK. Let us note that whenever $Y \subset D$, the assertion that $Y T Y$ is selfadjoint follows trivially.

AdDENDUM. After the completion of this work, a paper by James $P$. Williams [J. Math. Anal. Appl. 17 (1967), 214-220] appeared in which on p. 220 he attributes to Lumer the remark that if $A$ is a nonnegative bounded operator and if $B$ is selfadjoint, then $A^{1 / 2} B A^{1 / 2}$ is selfadjoint. This statement, seemingly more general than the above lemmas, is incorrect, as can be seen in the following counterexample. Let $B$ be an unbounded self adjoint operator, let $u$ be any element in JC which is not in the domain of $B$, and let $A$ be the projection operator onto $\operatorname{sp}\{u\}$. Then $A$ and $B$ satisfy the above hypotheses, but the operator $A^{1 / 2} B A^{1 / 2}$ is not selfadjoint. In fact, the domain of $A^{1 / 2} B A^{1 / 2}$ is $\{v \in \mathfrak{H C} \mid(u, v)=0\}$, which is not dense in $\mathfrak{H}$.

\section{REFERENCES}

1. A. Weinstein, The intermediate problems and the maximum-minimum theory of eigenvalues, J. Math. Mech. 12 (1963), 235-245. 
2. - An invariant formulation of the new maximum-minimum theory of eigenvalues, J. Math. Mech. 16 (1966) 213-218.

3. S. Goldberg, Unbounded linear operators: Theory and applications, McGrawHill, New York, 1966.

4. I. C. Gohberg and M. G. KreYn, Fundamental theorems on deficiency numbers, root numbers, and indices of linear operators, Uspehi Mat. Nauk 12 (1957), 43-188; English transl., Amer. Math. Soc. Transl. (2) 13 (1960), 185-264.

5. W. Stenger, The maximum-minimum principle for the eigenvalues of unbounded operators, Notices Amer. Math. Soc. 13 (1966), 731.

6. - On the variational principles for eigenvalues for a class of unbounded operators, J. Math. Mech. 17 (1968), 641-648.

The American University

\title{
ON AN ADDITIVE DECOMPOSITION OF FUNCTIONS OF SEVERAL COMPLEX VARIABLES
}

\author{
BY EDGAR KRAUT, STAVROS BUSENBERG AND WILLIAM HALL
}

Communicated by Maurice Heins, November 16, 1967

1. Introduction. Recent attempts (see [1] and the references in the same article) to extend the Wiener-Hopf technique for functions of a single complex variable to those of two or more complex variables have relied on a remark of Bochner's [2] that guarantees the required decomposition under suitable restrictions. Bochner's remark states that: if $f\left(z_{1}, \cdots, z_{n}\right), z_{j}=x_{j}+i y_{j}$, is analytic in a tube $T: \gamma_{i}<x_{i}<\delta_{i}$, $y_{i} \in(-\infty, \infty)$, and if $\int_{-\infty}^{\infty} \cdots \int\left|f\left(z_{1}, \cdots, z_{n}\right)\right|^{2} d y_{1} \cdots d y_{n}$ converges in $T$, then there exists in $T$ a decomposition $f=\sum_{i=1}^{2^{n}} f_{i}$, where each $f_{i}$ is analytic and bounded in an octant shaped tube $T_{i}$ containing the interior of $T$. Moreover, such a decomposition is unique up to additive constants. The uniqueness of the decomposition is not verified in [2] but reference is made to $\mathrm{H}$. Bohr's [3] corresponding result for functions of a single complex variable.

It is here shown that the uniqueness statement is false. However, the adjunction of the additional hypothesis that the $f_{i} \rightarrow 0$ when any one of the $x_{j} \rightarrow \infty$, in the tubes $T_{i}$, restores the uniqueness of the decomposition and justifies the use of the result in [2].

2. A counter-example. In the decomposition $f=\sum_{i=1}^{2 n} f_{i}, f_{1}$ is analytic and bounded in the tube $T_{1}: x_{i}>\gamma_{i}, y_{i} \in(-\infty, \infty), i=1,2$, $\cdots, n$, and $f_{2}$ is analytic and bounded in the tube $\left.T_{2}: x_{1}<\delta_{1}, x_{j}\right\rangle \gamma_{j}$, 